\title{
467 - A narrative review of the deprescribing process in patients with dementia
} Mariana Duarte Mangas, Gonçalo A. Santos, Catarina Pedro, Beatriz Jorge

Deprescribing, an integral component of a continuum of good prescribing practices, is the process of medication withdrawal or dose reduction to correct or prevent medication-related complications, improve outcomes, and reduce costs. Deprescribing can be a challenge in patients with dementia. In this narrative review we evaluate topics related with deprescribing: what constitutes deprescribing, the importance to deprescribing for patients with dementia, the potential benefits, barriers and enablers of deprescribing and the deprescribing process.

Patients with dementia often have multiple comorbidities and have complex medication regimens. Also, they face challenges with medication adherence because of the nature of the disease. Proper medication adherence is important to prevent progression of these comorbidities and decline in overall health. However, as dementia progresses, the risk of taking certain medications may outweigh the benefits. In addition, older people, especially those with physical and mental decline, tend to experience lower efficacy of these medications along with a higher risk of drug adverse effects. As with prescribing or continuing medications, deprescribing brings with it the potential for harm as well as benefit. Other barriers to deprescription include concerns from the patients or the family, worries and doubts from the physician and some issues related to each health system. Many challenges for its execution have been described.

Recent studies report benefits and safety in the prescription of patients with dementia, reinforcing the importance of considering prescription in the reevaluation of the patient. Advance care planning is the cornerstone of palliative care, and prescription should be considered in this process. 